\title{
Simulation of Cesium Injection and Distribution in RF-driven Ion Sources for Negative Hydrogen Ion Generation ${ }^{\text {a) }}$
}

\author{
R. Gutser, ${ }^{1}$ U. Fantz, ${ }^{1,2}$ and D. Wünderlich ${ }^{1}$ \\ ${ }^{1}$ Max-Planck-Institut für Plasmaphysik, EURATOM Association, Boltzmannstr. 2, 85748 Garching, Germany \\ ${ }^{2}$ Lst. f. Experimentelle Plasmaphysik, Universität Augsburg, 86135 Augsburg, Germany
}

\begin{abstract}
Cesium seeded sources for surface generated negative hydrogen ions are major components of neutral beam injection systems in future large-scale fusion experiments such as ITER. Stability and delivered current density depend highly on the cesium conditions during plasma-on and off phases of the ion source. The Monte Carlo code CsFlow3D was used to study the transport of neutral and ionic cesium in both phases. Homogeneous and intense flows were obtained from two cesium sources in the expansion region of the ion source and from a dispenser array, which is located $10 \mathrm{~cm}$ in front of the converter surface.
\end{abstract}

\section{THE RF-DRIVEN ION SOURCE}

A high-performance, large-area RF-driven negative hydrogen ion source for the ITER neutral beam injection system is being developed at the MPI für Plasmaphysik [1]. The ITER source must deliver $40 \mathrm{~A}$ of $\mathrm{D}^{-}$ions for 3600 seconds at a source pressure of $0.3 \mathrm{~Pa}$ while maintaining an electron to ion ratio $<1$ [2]. For the presently achieved negative deuterium ion current density of $200 \mathrm{~A} / \mathrm{m}^{2}$, the effective extraction area for the ITER source will be $2000 \mathrm{~cm}^{2}$. Negative ion production is done via the surface effect: positive and neutral hydrogen plasma particles hit a converter surface with a low work function, where they pick up one or more electrons. The ion source is operated with $\mathrm{D}_{2}$ and $\mathrm{H}_{2}$, while the latter is more often used because of the advantages in handling.

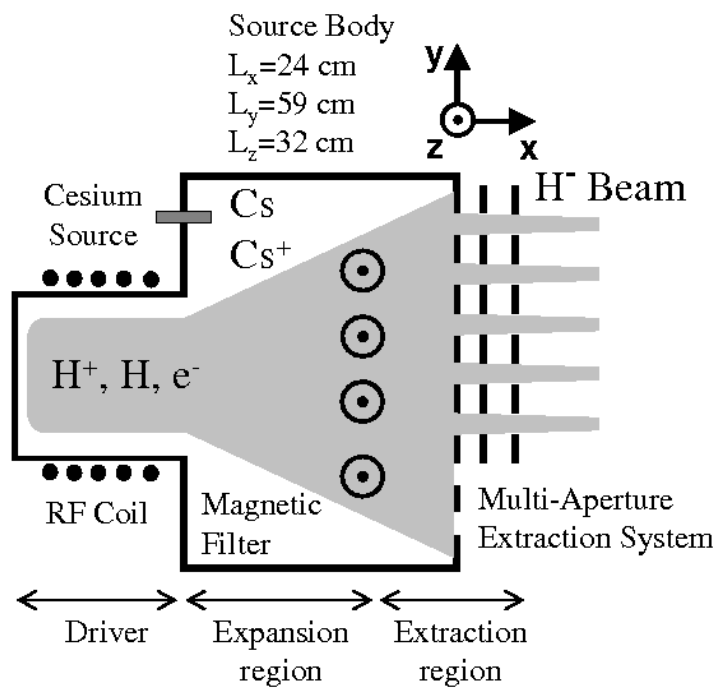

Fig. 1. Schematic view of the RF-driven ion source MANITU.
The IPP RF-driven ion source is divided into three parts: driver, expansion region and extraction region, as illustrated by figure 1 . The source is operated at a $\mathrm{H}_{2}$ density of $5 \times 10^{19} \mathrm{~m}^{-3}(1200 \mathrm{~K})$ and a RF power of up to $100 \mathrm{~kW}$. Electron densities of $\mathrm{n}_{\mathrm{e}}=2 \times 10^{18} \mathrm{~m}^{-3}$ and electron temperatures of $T_{e}>10 \mathrm{eV}$ are obtained at the driver exit [3][4]. In order to avoid significant negative ion destruction by electron stripping, a magnetic filter field of $7 \mathrm{mT}$ is used to reduce $T_{e}$ in the expansion region. Thus, typical $T_{e}$ of $2 \mathrm{eV}$ and $n_{e}$ of $2 \times 10^{17} \mathrm{~m}^{-3}$ are obtained in the extraction region [5]. The negative ion source test beds are operated in pulsed mode with typical pulse lengths between 3 and 10 minutes. A background vacuum of $10^{-6} \mathrm{mbar}$ is maintained in the plasma-off phases.

Cesium injection is done by a cesium source, which is located in the upper part of the backplate of the expansion region (see fig 1). The cesium from this supply has to be transported to the plasma facing surface (plasma grid) where an enhancement of the surface conversion rate is obtained by lowering the work function. Other cesiated surfaces do not contribute significantly to the extracted current density because of the short survival length (few $\mathrm{cm}[6]$ ) of the negative hydrogen ions. The intensity and homogeneity of the flow of neutral and ionic cesium during the plasma-on and off phases have high impact on the stability and performance of the negative ion production. A transport code for neutral cesium and cesium ions is being developed in order to understand and optimize the dynamics of cesium.

\section{THE TRANSPORT CODE CsFlow3D}

The CsFlow3D code simulates the transport of cesium in the plasma-on and off phases of the ion source. Geometry and parameters of the negative ion source test bed MANITU [7] having an extraction area of $204 \mathrm{~cm}^{2}$ were used in CsFlow3D. Because of the low ionization potential $(3.89 \mathrm{eV})$ of neutral cesium, it is necessary to consider ionization and recombination, which means the transition between neutrals and ions during the plasma phase. In the plasma-off phase, a free molecular flow regime is established and the atomic cesium particles follow straight lines. Transport during the discharge is dominated by collisions with background particles and the Lorentz force taking into account the electric and magnetic field topology [6] of the RF-driven ion source. Trajectories of statistically significant 
ensembles of $\mathrm{Cs} / \mathrm{Cs}^{+}$test particles are calculated within a background of field particle for a given density and temperature distribution. A constant distribution is used for the gas $\left(\mathrm{H}, \mathrm{H}_{2}\right)$ background and a parabolic one for the background of plasma $\left(\mathrm{H}^{+}, \mathrm{e}^{-}\right)$particles. These distributions were taken from measurements [3][4][5]. While frequent, small angle Coulomb collisions of $\mathrm{Cs}^{+}$ions with $\mathrm{H}^{+}$plasma particles are taken into account every time step [8], the remaining collision processes are treated by the path length estimator algorithm [9]. An overview of the used collision cross sections, which depend on the center of mass velocity $\mathrm{v}_{\mathrm{cm}}$ of the colliding particles, is given in table I.

An important part of the transport model is the surface model. Surface evaporation is modeled by a cosine redistribution of the evaporated cesium. Input data regarding the temperature dependent desorption coefficients of cesium in the plasma-off phase were taken from dedicated surface experiments, which have been performed in parallel to the code development [10]. Desorption coefficients of 0.1 and 0.3 were determined for sample temperatures of $26^{\circ} \mathrm{C}$ and $47^{\circ} \mathrm{C}$ [10]. Zero desorption was used during the discharge as a consequence of the formation of stable cesium hydride by reaction with the hydrogen background.

A constant flow of $10 \mathrm{mg} / \mathrm{h}$ from the cesium source during plasma-on and off phases was used corresponding to the experiment. Atomic cesium is able to leave the ion source through the extraction apertures, while the extraction potential inhibits the loss of ionic cesium during the discharge.

Additional cesium sources are formed by the discharge. Cesium is sputtered by $8 \mathrm{kV}$ backstreaming ions $\left(\mathrm{H}^{+}, \mathrm{H}_{2}^{+}\right)$ formed in the extracted ion beam and by hydrogen ions from the plasma inside the driver with a moderate energy $<55 \mathrm{eV}$. The sputtering yields were calculated by the TRIM code [11] assuming a surface binding energy of $1 \mathrm{eV}$. Thermally assisted desorption from the plasma facing side walls was estimated by means of an empirical scaling of the desorption rate with the power deposition on the plasma facing sidewalls. A mean value of $4 \times 10^{14} \mathrm{~cm}^{-2} \mathrm{sec}^{-1}$ is used for the calculation.

TABLE I. Collision processes considered in CsFlow3D.

\begin{tabular}{|c|c|c|}
\hline \hline Collision Process & Cross Section & $\begin{array}{c}\text { Data } \\
\text { Ref. }\end{array}$ \\
\hline$C s+H_{2} \rightarrow H_{2}+C s$ & Van der Waals $\frac{C^{\text {VdW }}}{v_{c m}^{2 / 5}}$ & {$[12]$} \\
$C s+e^{-} \rightarrow C s^{+}+2 e^{-}$ & Ionization & {$[13]$} \\
$C s^{+}+e^{-} \rightarrow C s+h v$ & Recombination & {$[14]$} \\
$\mathrm{Cs}^{+}+e^{-}($Wall $) \rightarrow C s$ & Langevin $\frac{C^{L}}{v_{c m}}$ & {$[15]$} \\
$\mathrm{Cs}^{+}+H_{2} \rightarrow H_{2}+C s^{+}$ & Coulomb $\frac{C^{C l}}{v_{c m}^{4}}$ & \\
$\mathrm{Cs}^{+}+H^{+} \rightarrow H^{+}+C s^{+}$ & & \\
& & \\
\hline
\end{tabular}

\section{RESULTS}

\section{A. Liquid Reservoir at Backplate}

The CsFlow3D code simulates the transport of cesium atoms and ions during cesium injection by a cesium source, which is connected to the backplate of the expansion region of MANITU (see fig 1). In the current source configuration, three nozzles of a single cesium source (S) point at the side walls (positive and negative $\mathrm{z}$-direction) and at the lower wall (negative y-direction) of the expansion region. Additionally, a design study for two cesium sources (D) considering a second cesium injection at the bottom (negative y-direction) of the expansion region was done. The nozzles of this additional source point at the side walls (positive and negative z-direction) and at the upper wall (positive $\mathrm{y}$-direction). Both calculations were performed for a constant and stable cesium injection rate of $10 \mathrm{mg} / \mathrm{h}$ in total. The cesium transport during the discharge was calculated for a previous plasma-off phase of $240 \mathrm{sec}$. and for total desorption inside the driver as a consequence of its high temperature. Vertical profiles of the flow of neutral cesium and cesium ions at the center of the plasma grid $(\mathrm{z}=0 \mathrm{~mm})$ are shown in fig. 2 and 3 for different desorption coefficients and source configurations (S/D). The fraction of neutral cesium during the discharge is negligible.

A neutral cesium flow of $10^{12} \mathrm{~cm}^{-2} \mathrm{sec}^{-1}$ (desorption $<0.9$ ) was calculated during the plasma-off phase, while the cesium ion flow of $10^{13} \mathrm{~cm}^{-2} \mathrm{sec}^{-1}$ during the discharge is significantly higher. This is an effect of the plasma related redistribution of condensed cesium from the ion source walls. Both cases show asymmetric flow profiles with a higher flow to the upper half of the plasma grid. Increasing the desorption coefficient by increasing the source wall temperatures causes higher flows during the plasma-off phase and a more symmetric profile during the discharge. This is a direct consequence of the more evenly distributed cesium on the walls during the preceding plasma-off phase at lower desorption coefficient. Improved homogeneity can also be obtained by using two cesium sources (dotted lines), which ensure a homogeneous cesium deposition. Operation with two cesium sources at $50^{\circ} \mathrm{C}$ wall temperature is preferable; because operation at high desorption coefficients $(0.9)$ causes high losses through the extraction apertures during the plasma-off phases.

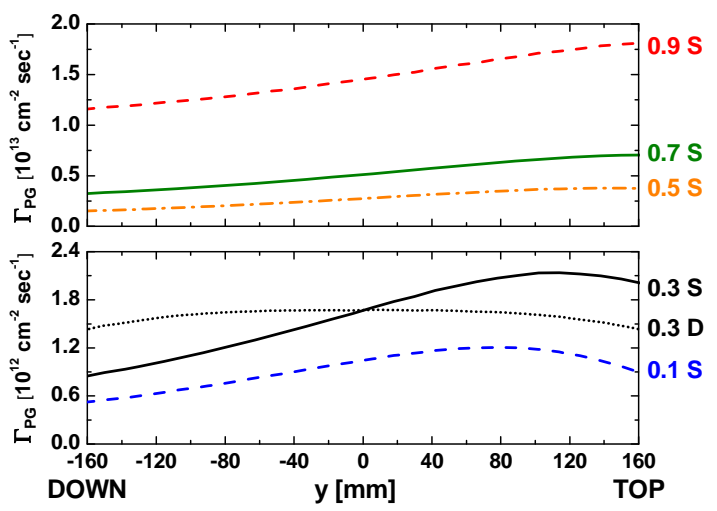

FIG. 2. Vertical flow profiles of neutral cesium on the plasma grid at $\mathrm{z}=0 \mathrm{~mm}$ considering different desorption coefficient (0.10.9 ) and oven configurations (D: Double /S: Single Oven). 


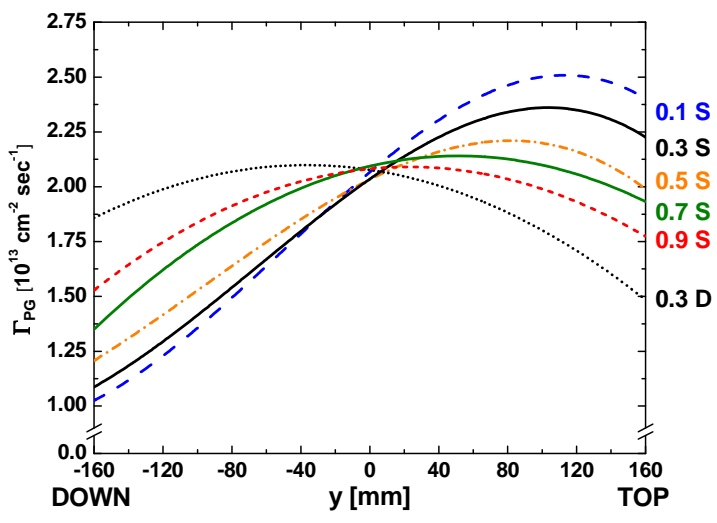

FIG. 3. Vertical flow profiles of cesium ions on the plasma grid at $\mathrm{z}=0 \mathrm{~mm}$ during the discharge considering different desorption coefficient (0.1-0.9) and oven configurations (D/S).

\section{B. Dispenser Array close to Plasma Grid}

A promising approach to obtain a stable and homogeneous cesium flow to the plasma grid is direct cesiation by an array of several small cesium (getter) sources oriented towards the plasma grid. Transport calculations for an arrangement of 8 dispensers at $\mathrm{y}=+/-5$ and $\mathrm{y}=+/-13 \mathrm{~cm}$ (for $\mathrm{z}=+/-5 \mathrm{~cm}$ ) with a cosine flow distribution and a total injection rate of $10 \mathrm{mg} / \mathrm{h}$ for several distances from the plasma grid were performed. Figure 4 shows a vertical profile of the cesium flow along four nozzles of the dispensers for a distance of 6,8 and $10 \mathrm{~cm}$ from the plasma grid during the plasma-on and off phases. The corresponding ionized fraction of the cesium flow is shown in fig. 5 . While the flow of neutral cesium during direct cesiation in the plasma-off phase is a factor of 10 higher than that in figure 2, comparable flow rates are achieved during the discharge. A distance of at least $10 \mathrm{~cm}$ for both phases will be required in order to obtain a homogeneous flow distribution over the plasma grid and an ion fraction of over $85 \%$. This high ionic fraction ensures that the extraction field keeps a high percentage of the cesium inside the ion source.

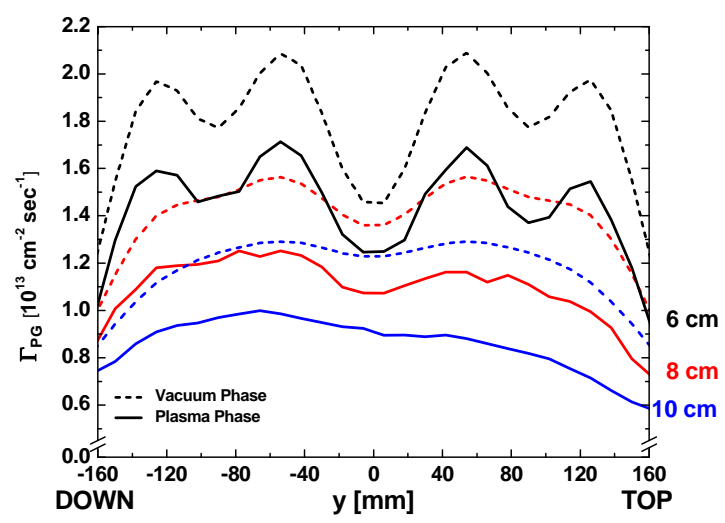

FIG. 4. Vertical flow profile of cesium neutrals and ions on the plasma grid at $\mathrm{z}=5 \mathrm{~cm}$ for the plasma-off phase (dashed line) and during the discharge (solid line) for different distances of the dispenser from the plasma grid.

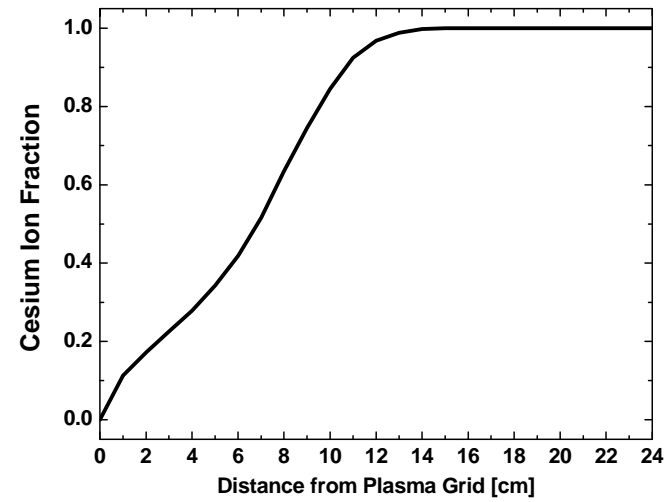

FIG. 5. Fraction of ionic cesium versus the position of the cesium dispenser array in front of the plasma grid.

\section{CONCLUSION}

Transport calculation for different cesium source designs and source wall conditions were performed with the CsFlow3D code. Calculations for a single cesium source in the upper part of the ion source result in an inhomogeneous flow profile over the plasma grid. The increase of the wall desorption coefficient by increasing the wall temperatures results in an increase of the flow during the plasma-off phase and a more homogeneous flow profile during discharge. An improvement of the homogeneity can also be obtained by using two cesium sources, which are preferable regarding the cesium losses through the apertures during the plasma-off phase. Calculation of a dispenser array, which is close to the plasma grid, show that a distance of at least $10 \mathrm{~cm}$ from the plasma grid is required to obtain a homogeneous profile. This direct cesiation does not rely on the redistribution of the cesium supplies on the source walls during the discharge which might be beneficial for stability and control.

\section{Acknowledgements}

This work was (partly) supported by a grant from the European Union within the framework of EFDA. The authors are solely responsible for the content.

\section{References}

${ }^{1}$ E. Speth, H.D. Falter, P. Franzen, U. Fantz, M. Bandyopadhyay, S. Christ, A. Encheva et al., Nucl. Fusion 46, S220 (2006)

${ }^{2}$ ITER Technical Basis 2002, ITER EDA Documentation Series No 24 (Plant Description Document, section 2.5.1), IAEA Vienna

${ }^{3}$ A. Tanga, and M. Bandyopadhyay, Appl. Phys. Lett., 84, 182 (2004)

${ }^{4}$ S. Dietrich, Ringberg 2007, private communication

${ }^{5}$ U. Fantz, H.D. Falter, P. Franzen, D. Wünderlich, M. Berger, A. Lorenz,

W. Kraus et al., Nucl. Fusion 46, S297 (2006)

${ }^{6}$ R. Gutser, D. Wünderlich, U. Fantz and the NNBI-Team, Plasma Phys. Control. Fusion 51, 045005 (2009)

${ }^{7}$ W. Kraus, H.D. Falter, U. Fantz, P. Franzen, B. Heinemann, P. McNeely, R. Riedl and E. Speth, Rev. Sci. Instrum. 79, 02 C108 (2008)

${ }^{8}$ S. Ma, R. Sydora and J. Dawson, Comp. Phys. Com. 77, 190 (1993)

${ }^{9}$ C. Birdsall, IEEE Trans. on Plasma Sci. 19, 65 (1991)

${ }^{10}$ U. Fantz, R. Gutser and C. Wimmer, Rev. Sci. Instrum. 81, 1 (2010)

${ }^{11}$ W. Eckstein and J. P. Biersack, Appl. Phys. A 38, 123 (1985),

${ }^{12}$ E. Rothe and R. Bernstein, J. Chem. Phys. 31, 1619 (1959)

${ }^{13}$ M. Lukomski, Phys. Rev. A 74, 032708 (2006)

${ }^{14}$ I. H. Hutchinson, Principles of Plasma Diagnostics, (Cambridge University Press, Cambridge UK, 2002)

${ }^{15}$ D. Lide, CRC Handbook of Chemistry and Physics 89th ed. (CRC, Cleveland OH, 2008) 